\title{
Illumination based Sub Image Histogram Equalization: A Novel Method of Image Contrast Enhancement
}

\author{
Sangeeta Rani \\ Deptt of ECE, \\ IGDTUW, Delhi
}

\author{
Ashwini Kumar \\ Deptt of ECE, \\ IGDTUW, Delhi
}

\author{
Kuldeep Singh \\ Central Research Lab \\ BEL, Ghaziabad
}

\begin{abstract}
A novel Illumination based Sub-Image Histogram Equalization (ISIHE) method for contrast enhancement for low illumination gray scale images is presented in this paper. As the main crux of paper, illumination thresholds are computed and used to divide the original image into subimages of different intensity levels. To control the enhancement rate, the histogram is clipped using a threshold value that represents the average number of grey level occurrences in the image. Each individual sub histogram is equalized independently and all sub images are integrated into one complete image for analysis as a final step. The experimental results are compared with other Histogram Equalization (HE) methods and ISIHE has shown promising results.
\end{abstract}

\section{General Terms}

Image Processing, Histogram Equalization

\section{Keywords}

Image Contrast Enhancement, Image Illumination Threshold, Histogram Equalization

\section{INTRODUCTION}

Remarkable enhancements in the VLSI, Signal and Image Processing technology over the decades has enabled the major revelation in the consumer electronics be it televisions, camera and mobile or cell-phones. Now days even a lowest model of any mobile have digital camera in built and now a day's mobile phones have been widely used to take pictures in daily life. Since Mobile phones have limited hardware capability for digital photography hence post processing of images using software tools is highly required to improve the quality of acquired image. In view of this contrast enhancement and brightness preservation are two prime focus areas for researchers in the field of consumer electronics products. Image enhancement improves the appearance of image and enhances the finer details of image having low luminance. The image enhancement techniques can be broadly divided into two categories- Transform domain and spatial domain [1]. First category includes the technique that operates on frequency transform of an image whereas the techniques like contrast enhancement in the second category operates directly on the pixel level of the image.

Histogram equalization (HE) is widely used for contrast enhancement in a variety of applications due to its simple function, ease of implementation and effectiveness [1]. Examples include medical image processing and radar signal processing. The idea behind Histogram Equalization (HE) is to flatten the probability distribution and stretching the dynamic range of gray levels, which in result improves the overall contrast of the image [2].
HE utilizes the cumulative density function (cdf) of image for mapping the gray levels of original image to the enhanced image. HE is not suitable for most consumer electronics applications such as TV, Cameras etc., as it tends to change the mean brightness of the image to the middle level of the gray level range, which in turn produces annoying artifacts and intensity saturation effects. Various methods have been suggested in literature to overcome the above-mentioned shortcomings. But enhancement for low illumination images is still needs more attention. In this paper, we propose a novel method of image contrast enhancement named illumination based Sub-Image Histogram Equalization (ISIHE), which is very effective for low illumination gray scale images and preserves entropy along with control on enhancement rate. The authors believe that the proposed ISIHE method shall achieve not only the objective of entropy maximization but also provide controlled enhancement. The authors also believe that the proposed method shall be a better approach for image enhancement of low illuminated images.

This paper is organized in sections as follows: Section 2 describes the existing literature and research work for HE based image enhancement technique. Section 3 describes the proposed ISIHE method. Section 4 gives experimental results. Section 5 presents the conclusion of the paper.

\section{RELATED WORK}

As described in the introduction, $\mathrm{HE}$ is the one of the renowned methods for enhancing the contrast of given images in accordance with the spatial distribution of an image[2]. A wide variety of $\mathrm{HE}$ based image enhancement techniques are available in literature. This section shall provide an insight into the work done by researchers for image contrast enhancement based on HE. The first method named Brightness preserving bi histogram equalization (BBHE) was proposed by Kim et al.[2] in 1997. It preserves the mean brightness of image while improving the contrast. BBHE divides the histogram in two parts based on the input mean brightness and equalizes the two sub histograms independently. In 1998, another method named Multi Peak Histogram Equalization with Brightness Preserving (MPHEBP) has been proposed by Wongsritong et al.[3]. In this method, the input histogram of the image is smoothed and divided based on the local maxima. It improves the mechanism of preserving brightness of the image while improving the contrast. Authors claimed that the performance of MPHEBP is better than BBHE in terms of maintenance of mean brightness. In 1999, a method named Dualistic Sub Image Histogram Equalization (DSIHE) has been proposed by

Wang et al.[4] and claimed it to be better than BBHE in terms of preservation of brightness and average information content (entropy) of an image. DSIHE divides the histogram in two 
sub histograms containing equal number of bins and the division is based on median value instead of mean brightness.

Another method named minimum mean brightness error bihistogram equalization (MMBEBHE) which is an improvement over BBHE for preserving the mean brightness "optimally" has been introduced by Chen et al.[5] in 2003. The method calculates the absolute mean brightness error (AMBE) for gray levels 0 to L-1 and bisects the histogram based on the intensity value, which yields minimum AMBE. Chen et al. [6] in 2003 has also proposed another approach known as recursive mean-separate histogram equalization (RMSHE) that recursively performs the BBHE by dividing the histogram into two parts on the basis of average input brightness followed by application of BBHE to each sub histogram independently.

In 2003, Yang et al.[7] proposed a simple enhancement rate control mechanism, Bin Underflow and Bin Overflow (BUBO) that controls the rate of enhancement by putting constraints on the maximum and minimum gradient of the mapping function. HE can enhance the contrast to variable rates with this enhancement rate control mechanism. It also performs various image processing tasks such as black/white level stretch or automatic brightness control.

In order to enhance the main objects and to suppress the background for infrared images Wang et al.[8] in 2006 proposed Self-Adaptive Plateau Histogram Equalization (SAPHE) method. In SAPHE, histogram of the image is filtered with median filter to reduce the fluctuations, to remove the empty bins within the histogram and to find the local maximum value and global maximum value of histogram for plateau threshold value.

Another method which is similar to RMSHE, is proposed by Sim et al.[9] in 2007. This algorithm performs the division of histogram based on median value of brightness instead of mean brightness and termed as Recursive Sub-Image Histogram Equalization (RSIHE). Finding the optimal value of iteration factor is a big challenge for producing significant enhancement results both in RMSHE and RSIHE methods.

In 2007, Wadud et al.[10] has introduced a method of Dynamic Histogram Equalization (DHE) that was developed for elimination of domination of higher histogram components on lower ones in the original histogram. The method controls the grey level stretching in image so that the reasonable enhancement of the image features can be achieved by using local minima separation of histogram. DHE ignores the mean brightness preservation and tends to intensity saturation artifacts. To overcome the limitations of the DHE, Ibrahim et al. [11] in 2007 has introduced Brightness Preserving Dynamic Histogram Equalization (BPDHE). This method is basically a combination of DHE and Multi Peak Histogram Equalization with Brightness Preserving (MPHEBP). BPDHE has shown better performance as compared to MPHEBP and better preservation of mean brightness in comparison to DHE.

To yields images with natural appearances, at the cost of contrast enhancement, Menotti et al.[12] in 2007 has proposed Minimum Within-Class Variance Multi-Histogram Equalization (MWCVMHE) and Minimum Middle Level Squared Error Multi Histogram Equalization (MMLSEMHE) techniques. MWCVMHE divides the original histogram into multiple sub-histograms by minimizing within-class variance and then applies histogram equalization to each individual sub-histogram separately. In MMLSEMHE, Otsu threshold has been used for selecting the separation points for obtaining the individual sub-histograms and then each individual sub histogram is equalized independently. MMLSEMHE is computationally more complex because it estimates the optimal number of sub-histograms from all possible subhistograms to minimize certain discrepancy functions [13]. In both the methods the contrast enhancement is less intensive but the brightness is preserved to a maximum extent.

A new method of contrast enhancement for controlling noise amplification and preserving the original brightness of the image has been introduced by Kim et al. [14] in 2008. The method is termed as Gain-Controllable Clipped Histogram Equalization (GC-CHE). GC-CHE is a different way of looking at BBHE and RMSHE methods. In GC-GHE method, the histogram of the image is clipped based on clipping threshold and the clipped portion is then re-distributed to the entire dynamic range by locally regulating the clipping gain. In this method, the contrast elevation ratio is adjusted to solve the noise amplification problem as per input image and compensate contrast using the gain control method.

A method known as Bi-Histogram Equalization Plateau Limit (BHEPL) has been proposed by Ooi et al. [15] in 2009. This method basically is the fusion of the BBHE and clipped histogram equalization approaches. BHEPL decomposes the input image into two sub-images by using mean brightness of the image followed by clipping of sub-histograms by using the plateau limit as the mean of the number of intensity occurrence and equalization of each sub histogram independently. BHEPL method avoids excessive enhancement and over amplification of noise in the image. A similar approach has been proposed by Ooi et al.[16] in 2010 that categorizes the original histogram into four sub-histograms based on the median of the input image. This method is based on clipping of histogram and termed as Quadrants Dynamic Histogram equalization (QDHE). The resultant subhistograms are clipped according to the mean of intensity occurrence of the input image before new dynamic range is assigned to each sub-histogram and are equalized individually. QDHE is most robust method to extract the details of the low contrast images.

The methods proposed in [14][15][16] controls maximum value of histogram by clipping histograms higher than the pre specified threshold. These methods provide different approach for determination of clipping threshold. Liang et al., [17] in 2012, proposed Double Plateaus Histogram Equalization (DPHE) for infrared image enhancement. In this method, upper and lower threshold values could be calculated by searching local maximum and predicting minimum gray interval. The value of upper threshold is set to be $20-30 \%$ of the total pixels, while the lower threshold value is set to be 5$10 \%$ of it. The upper threshold is utilized in the algorithm for preventing over-enhancement of background noise with typical gray levels, and the lower threshold is set for protecting detailed information with fewer pixels from being combined.

Singh and Kapoor [18] presents a novel Exposure based SubImage Histogram Equalization (ESIHE) method for contrast enhancement for low exposure gray scale image. Singh and Kapoor [19] also proposed Median-Mean based sub-image clipped histogram equalization MMSICHE algorithm for image enhancement, which firstly performs histogram partition based on median intensity and then divides each subhistograms based on mean intensity. Although a wide variety of methods and techniques are available in literature to handle specific issues in contrast enhancement, but enhancement for 
low illumination images is still less explored area. Therefore a novel method termed as Illumination based Sub-Image Histogram Equalization (ISIHE) is proposed for low illumination gray scale images. The proposed method is very effective for such images and preserves entropy along with control on enhancement rate. The authors believe that the ISIHE method achieves both the objectives of entropy maximization and control on over enhancement. We also believe that the proposed approach is better for image enhancement specifically for under illuminated images

\section{PROPOSED METHOD}

The images which have histogram bins more concentrated towards lower part or the darker gray levels possess low intensity illumination whereas images having histogram bins concentrated towards higher part or the brighter part possess high intensity illumination. Based on the intensity of illumination, images can be broadly classified as under or over illuminated image. In this section, a novel method of image contrast enhancement based on Illumination categorization and histogram equalization is presented. The method termed as Illumination based Sub-Image Histogram Equalization (ISIHE). The proposed method of ISIHE is shall consists of three steps, namely Illumination thresholds calculation, Histogram Clipping and Histogram Sub division $\&$ Equalization. These three steps are explained as follows:

\subsection{Calculation of Illumination Threshold Parameter}

An Illumination Threshold parameter $\left(I_{T}\right)$ is defined to denote the measure of illumination intensity of the target image. $I_{T}$ is used to divide the image into under and over illuminated sub images .The normalized range of illumination value is [0-1]. If the value of illumination for a particular image is more than 0.5 and tends towards 1 , it means that the image has majority of over illuminated region where as if it is less than 0.5 and tends towards 0 then image contains majority of under illuminated regions. In both cases image contains poor contrast and need contrast enhancement. $I_{T}$ is defined in equation (1):

$$
I_{T}=L \frac{\sum_{i, j}\left[X(i, j)-\operatorname{mean}\left(X(i, j)_{3 X 3}\right)\right]^{2}}{\sum_{i, j} 1 / 9 \sum_{r=i-1}^{i+1} \sum_{s=j-1}^{j+1}[X(i, j)-X(r, s)]^{2}}
$$

This parameter attains a value of greater or lesser than $L / 2$ (gray level) for illumination value lesser or greater than 0.5 respectively for an image having dynamic range 0 to $\mathrm{L}$.

\subsection{This Histogram Bisection and Clipping}

The original histogram of the target image is bisected based on illumination threshold parameter value as $I_{T}$ calculated in equation (1) followed by clipping of the histogram. The idea behind histogram clipping is to prevent over enhancement leading to natural appearance of image. The histogram bins having the value greater than the clipping threshold $\left(C_{T}\right)$ are limited to the threshold [15]. The clipping threshold is calculated as an average number of grey level occurrences. The formula for clipping threshold $\left(C_{T}\right)$ is defined by equation (2) and (3).

$$
\begin{aligned}
& C_{T}=\frac{1}{L} \sum_{l=1}^{L} H(l) \\
& H_{c}(l)=C_{T} \text { for } H(l) \geq C_{T}
\end{aligned}
$$

Where $H(l)$ and $H_{c}(l)$ are the original and clipped histogram respectively.
The histogram is first bisected based on illumination threshold value $I_{T}$ as calculated in (1). The Histogram Sub Division process results in two sub images ranging from gray level 0 to $I_{T}$ and $I_{T}+l$ to $L-l$ respectively. $\mathrm{P}_{\text {Lower }}(l)$ and $\mathrm{P}_{\text {Upper }}(l)$ are corresponding probability density function $(p d f)$ of sub images

$$
\begin{gathered}
\mathrm{P}_{\text {Lower }}(l)=H_{c}(l) / N_{\text {Lower }} \quad \text { for } 0 \leq l \leq I_{T} \\
\mathrm{P}_{\text {Upper }}(l)=H_{c}(l) / N_{\text {Upper }} \text { for } I_{T}+1 \leq l \leq L-1
\end{gathered}
$$

Where $N_{\text {Lower }}$ and $N_{\text {Upper }}$ are total number of pixels in sub images. $\quad C_{\text {lower }}(l)$ and $C_{\text {Upper }}(l)$ are corresponding cumulative distribution function $(c d f)$ of individual sub images

$$
\begin{aligned}
& C_{\text {lower }}(l)=\sum_{l=0}^{I_{T}} \mathrm{P}_{\text {Lower }}(l) \\
& C_{\text {Upper }}(l)=\sum_{l=I_{T}+1}^{L-1} \mathrm{P}_{\text {Upper }}(l)
\end{aligned}
$$

The next step of ISIHE is to equalize all the four sub histograms individually. The transfer functions used for sub image histogram equalization can be defined as:

$$
\begin{aligned}
& T_{\text {Lower }}=I_{T}+C_{\text {Lower }} \\
& T_{\text {Upper }}=\left(I_{T}+1\right)+\left(L-I_{T}+1\right) C_{\text {Upper }}
\end{aligned}
$$

The output image is computed as integration of both histogram equalized sub images.

\subsection{Algorithm of ISIHE}

- Compute histogram $H(l)$ of target image

- Compute the value of Illumination threshold parameter $I_{T}$

- Compute the clipping threshold $C_{T}$ and clip the histogram $H(l)$ to $H_{C}(l)$ using $C_{T}$

- Divide the clipped histogram into two sub histograms using the threshold parameter $\mathrm{I}_{\mathrm{T}}$.

- Apply HE on individual sub histograms

- Integrate the sub images into single image

\section{EXPERIMENT RESULTS}

This section presents the experimental results of proposed ISIHE method. The proposed method is applied to various images. For performance evaluation three images viz Aircraft, Field and Mosque images as shown in figure 1,2 and 3 have been considered as the targets images. The results obtained from the ISIHE method have been compared with the results of existing methods of histogram equalization such as BBHE, MMBEBHE, DSIHE, RMSHE and RSIHE applied to same targets images. Entropy (Shannon Entropy) as defined in (10)have been used as one of the metric for image quality measure. It is considered as the measure of richness of details in the image andusually measured in bits. Higher the value of the entropy of an image, larger the information content in the image which is always desirable.

$$
\text { Entropy }=\sum_{k=0}^{L-1} P d f(k) \log P d f(k)
$$

Where $\operatorname{Pdf}(\mathrm{k})$ is pdf of a given image at intensity level $\mathrm{k}$ and $\mathrm{L}$ is total number of gray levels in the image. 


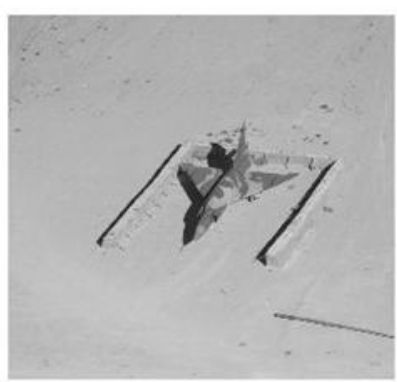

(a)

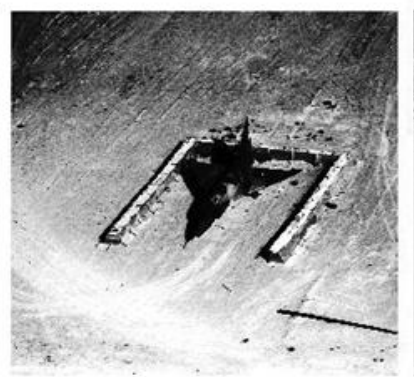

(e)

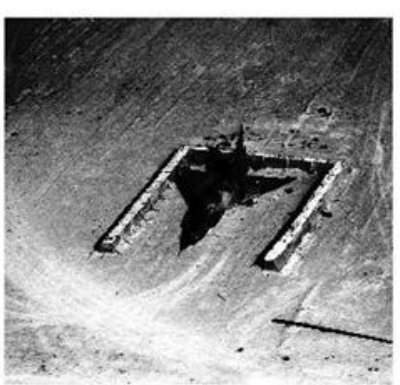

(b)

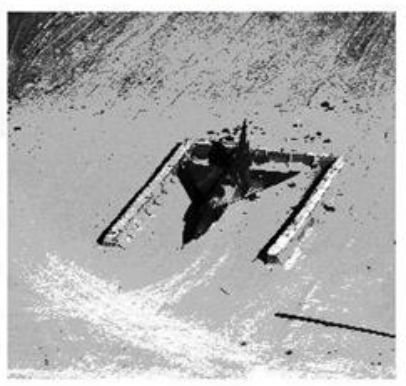

(t)

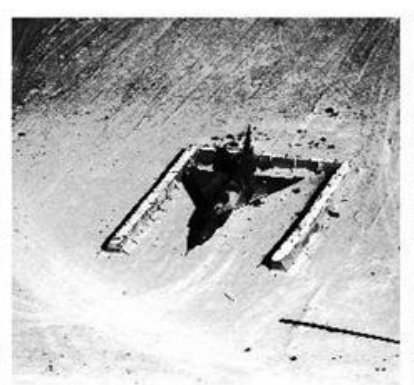

(c)

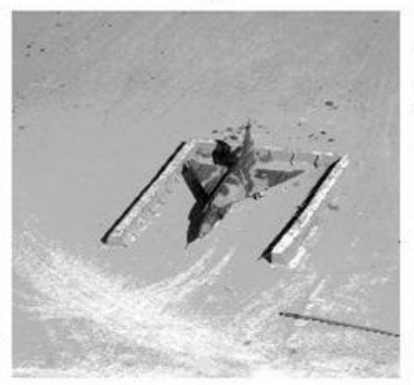

(g)

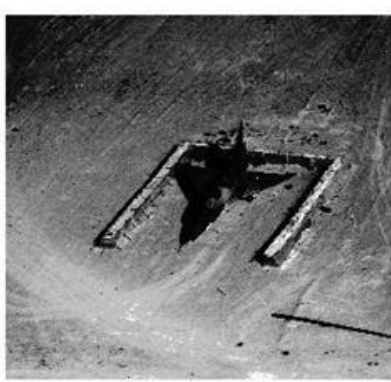

(d)

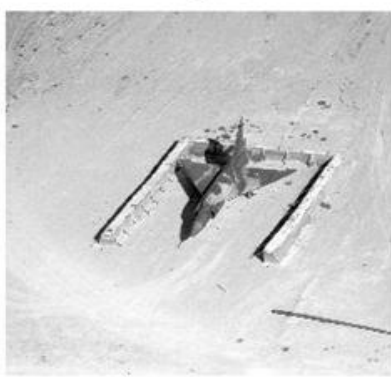

(h)

Fig 1: Visual Inspection of Aircraft image (a) Original, (b) HE, (c) BBHE, (d) MMBEBHE, (e) DSIHE, (f) RSIHE, (g) RMSHE and (h) ISIHE

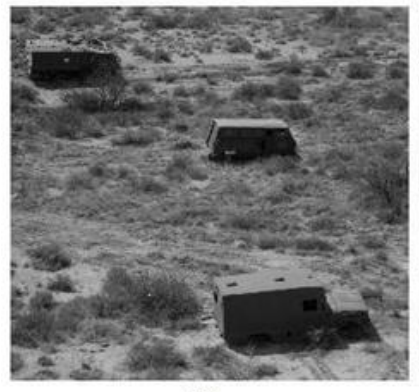

(a)

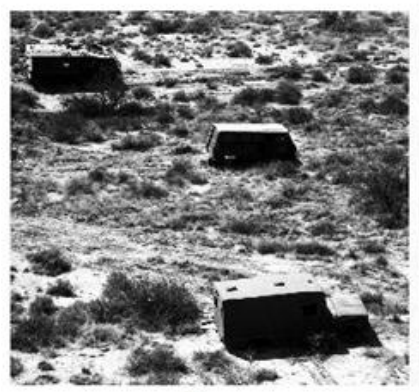

(e)

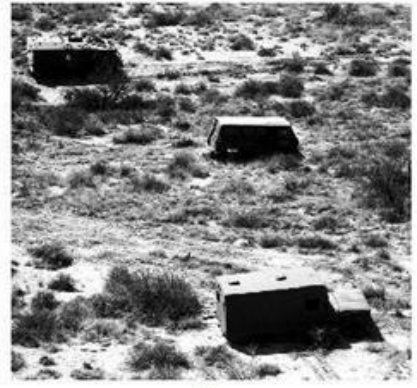

(b)

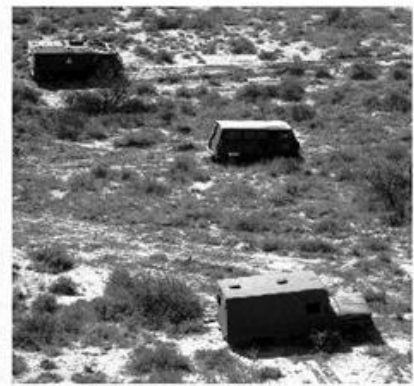

(i)

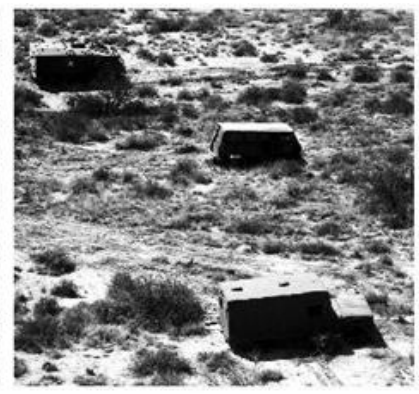

(c)

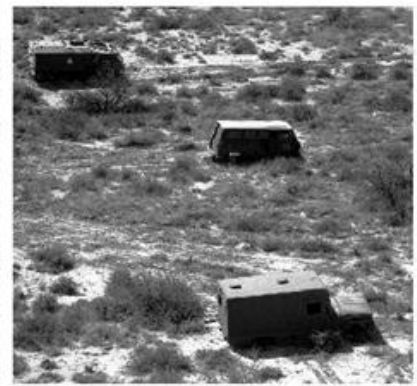

(g)

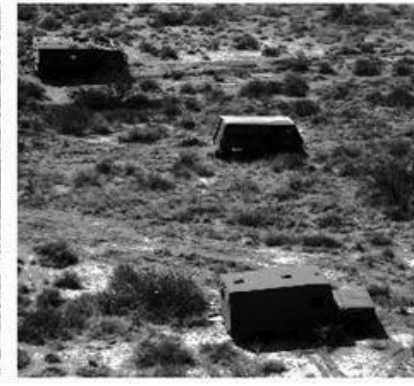

(d)

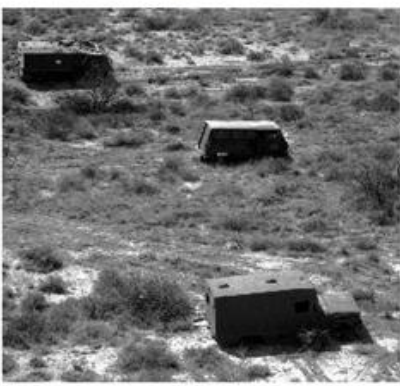

(h)

Fig 2: Visual Inspection of Field image (a) Original, (b) HE, (c) BBHE, (d) MMBEBHE, (e) DSIHE, (f) RSIHE, (g) RMSHE and (h) ISIHE 


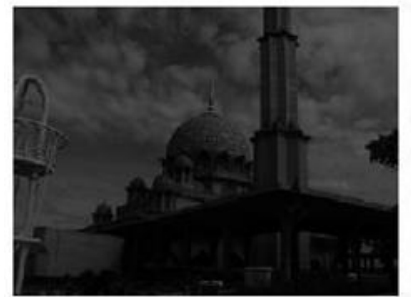

(a)

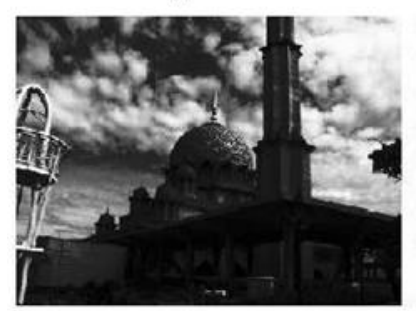

(e)

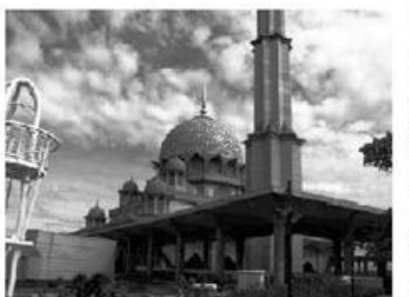

(b)

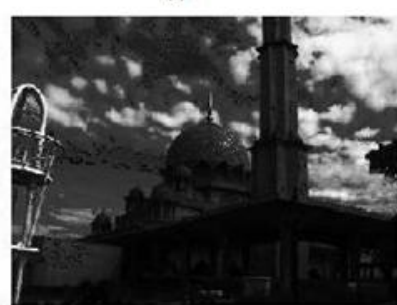

(f)

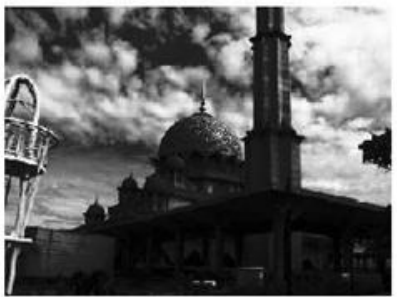

(c)

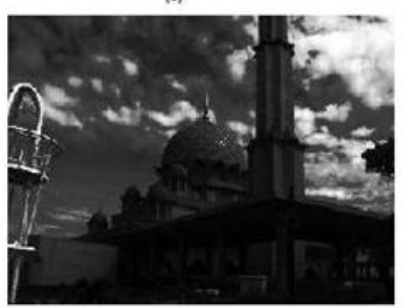

(g)

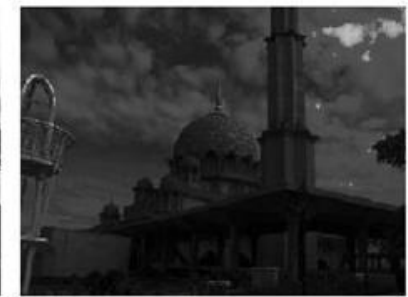

(d)

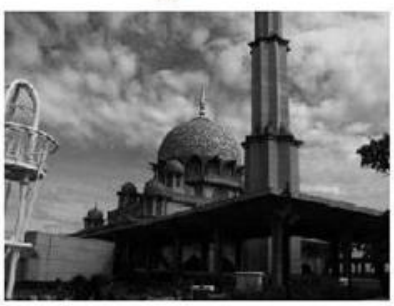

(h)

Fig 3: Visual Inspection of Mosque image: (a) Original, (b) HE, (c) BBHE, (d) MMBEBHE, (e) DSIHE, (f) RSIHE, (g) RMSHE and (h) ISIHE

Table-1 Resultant Entropies of different methods

\begin{tabular}{|l|l|l|l|l|l|l|l|l|}
\hline Images & Original & HE & BBHE & MMBEBHE & DSIHE & RSIHE(r=2) & RMSHE(r=2) & ISIHE \\
\hline Mosque & 6.26 & 5.83 & 6.11 & 6.06 & 6.09 & 6.08 & 6.10 & 6.19 \\
\hline Field & 6.56 & 5.96 & 6.46 & 6.41 & 6.46 & 6.52 & 6.49 & 6.54 \\
\hline Aircraft & 4.00 & 3.75 & 3.90 & 3.86 & 3.87 & 3.95 & 3.94 & 3.97 \\
\hline Average & 5.60 & 5.18 & 5.49 & 5.44 & 5.47 & 5.51 & & 5.51 \\
\hline
\end{tabular}

\subsection{Entropy based evaluation}

In order to evaluate the performance of the ISIHE method, discrete entropy is computed for each target image using different HE methods as mentioned in previous section. The resultant entropies as obtained using different HE methods when applied on target images are given in the table-1. The analysis of the results presented in the table- 1 indicates that the proposed method produces the highest entropy value for all the imagesas compared to other HE methods thereby proves to be the best suited method for identifying the information richness of the image.

As per table-1, the entropy values as obtained from the ISIHE method are nearly equal to the entropy of original image for all the three images whereas the entropy values for BBHE,MMBEBHE and MMBEBHE are very less in comparison to the entropy of original image. Another method DSIHE, which is claimed to be better in terms of average information content of image is also having entropy values lesser than the proposed method.The average of entropy produced by ISIHE method for all images is 5.39 that is very close to average entropy (5.43) for original images, however average entropy of other methods is much smaller in comparison with original image. The entropy closer to original image guarantees extraction of maximum information content of the image.

Since the proposed method produces the highest entropy value amongst all the methods which is also closer to the original value of entropy of the image, hence it proves to be the bestsuited method for bringing out the information contents of the image.

\subsection{Visual Inspectionbased evaluation}

Visual inspection is a necessity for judgment of annoying artifacts, over and unnatural enhancement.Table- 1 shows the quantitative assessment whereas figure 1-3 provides visual qualitative assessment. The visual assessment results are effective quality measures to judge the performance of contrast enhancement algorithm. The analysis of visual results from Figure 1-3 indicates that the results obtained from ISIHE method are promising in terms of contrast enhancement and visual appearance. 


\section{CONCLUSION}

This paper presents new method of contrast enhancement by sub division of image based on illumination parameter and histogram equalization of individual sub images. Illumination based division of image and $\mathrm{HE}$ of sub images has proved to be a very effective technique for enhancing under illuminated images. ISIHE compensates for low illumination by introducing higher gray levels in sub image so that after individual histogram equalization process over all the illumination value increases. From the quantitative and qualitative measures and evaluation, it is well observed that ISIHE method is well suited for under illuminated images(illumination value less than 0.5 ) and best in terms of entropy (richness in information) in comparison to other methods. The proposed method can be applied recursively to improve the performance of enhancement of low illuminated images as part of future scope of work.

\section{REFERENCES}

[1] Gonzalez, R.C., Woods, R.E., 2002. Digital Image processing, second ed. Prentice Hall.

[2] Kim, Y.T., 1997. Contrast enhancement using brightness preserving bi-histogram equalization. IEEE Trans. Consumer Electron. 43 (1), 1-8.

[3] Wongsritong, K., Kittayaruasiriwat, K.., F., Cheevasuvit , K., and Somboonkaew, A., "Contrast Enhancement using Multipeak Histogram Equalization with Brightness Preserving", IEEE Asia-Pacific Conference on Circuits and Systems, (1998).

[4] Wang, Y., Chen, Q., Zhang, B.M., 1999. Image enhancement based on equal area dualistic sub-image histogram equalization method. IEEE Trans. Consumer Electron. 45 (1), 68-75.

[5] Chen, S.D., Ramli, A.R., 2003. Minimum mean brightness error bi-histogram equalization in contrast enhancement. IEEE Trans. Consumer Electron. 49 (4), $1310-1319$

[6] Chen, S.D., Ramli, A.R., 2003. Contrast enhancement using recursive mean-separate histogram equalization for scalable brightness preservation. IEEE Trans. Consumer Electron. 49 (4), 1301-1309.

[7] Yang, S., Oh, J. H. and Park, Y., 2003. Contrast Enhancement using Histogram Equalization with Bin Underflow and Bin Overflow", International Conference on Image Processing ICIP-2003, vol. 1, 881-884.

[8] Wang, B. J., Liu , S.Q., Li, Q. and Zhou, H.X., 2006. A real-time Contrast Enhancement Algorithm for Infrared
Images based on Plateau Histogram", Infrared Physics \& Technology, 48, 77-82.

[9] Sim, K.S., Tso, C.P., Tan, Y.Y., 2007. Recursive subimage histogram equalization applied to gray scale images. Pattern Recogn. Lett. 28 (10), 1209-1221.

[10] Wadud, M. A., Kabir, M. H., Dewan, M.A.A., Chae, O., 2007. A Dynamic Histogram Equalization for Image Contrast Enhancement, IEEE Trans. Consumer Electron., 53, 593-600.

[11] Ibrahim, H., Kong, N. S. P., 2007. Brightness Preserving Dynamic Histogram Equalization for Image Contrast Enhancement, IEEE Trans. Consumer Electron., $53,1752-1758$

[12] Menotti, D., Najman, L., Facon , J. and Araujo, A. D. A., 2007 . Multi-Histogram Equalization Methods for Contrast Enhancement and Brightness Preserving, IEEE Transactions on Consumer Electronics, 53(3), 11861194.

[13] Sengee, N. and Choi, H. K., 2008. Brightness preserving weight clustering histogram equalization, IEEE Transactions on Consumer Electronics, 54(3), 1329. 1337.

[14] Kim, T., Paik, J., 2008. Adaptive contrast enhancement using gain-controllable clipped histogram equalization. IEEE Trans. Consumer Electron. 54 (4), 1803-1810.

[15] Ooi, C.H., Kong, N.S.P., Ibrahim, H., 2009. Bihistogram equalization with a plateau limit for digital image enhancement. IEEE Trans. Consumer Electron. 55 (4), 2072-2080.

[16] Ooi, C.H., Isa, N. A. M., 2010. Adaptive Contrast Enhancement Methods with Brightness Preserving, IEEE Trans. on Consumer Electron., 56, 2543 - 2551.

[17] Liang, K., Ma, Y., Xie , Y., Zhou, B. and Wang, R., 2012. A new adaptive contrast enhancement algorithm for infrared images based on double plateaus histogram equalization, Infrared Physics \& Technology, 55, 309315 .

[18] Singh, K., Kapoor, R., 2014. Image enhancement using Exposure based Sub Image Histogram Equalization, Pattern Recognition Letters , 36, 10 - 14

[19] Singh, K., Kapoor, R., 2014. Image enhancement via Median-Mean Based Sub-Image-Clipped Histogram Equalization, Optik, 125,4646-4651 